\title{
Can alpha-1-acid glycoprotein affect the outcome of treatment in a cancer patient?
}

\author{
Pitekova $\mathrm{B}^{1}$, Uhlikova $\mathrm{E}^{1}$, Kupcova $\mathrm{V}^{2}$, Durfinova $\mathrm{M}^{1}$, Mojto $\mathrm{V}^{2}$, Turecky L \\ Institute of Medical Chemistry, Biochemistry and Clinical Biochemistry, Faculty of Medicine, \\ Comenius University, Bratislava, Slovakia. ladislav.turecky@fmed.uniba.sk
}

\begin{abstract}
Alpha-1-acid glycoprotein (AGP) is a glycoprotein found in the alpha-1 globulin fraction of human plasma proteins. AGP is an important representative of acute-phase proteins. Although numerous articles have been devoted to AGP, its exact biological function remains obscure. AGP levels increase with a number of diseases. One of them is a tumor disease. In our paper, we discuss the role of increased AGP levels in cancer patients. We deal with the role of AGP as a drug-binding protein and its effect on the efficacy of chemotherapy in oncological patients. Other problems that are discussed in our paper include the role of AGP as an immunomodulatory protein and its relationship to angiogenesis because angiogenesis plays an important role in the progression of cancer (Ref. 57). Text in PDF www.elis.sk.

KEY WORDS: alpha-1-acid glycoprotein, orosomucoid, cancer, disease marker, immunomodulatory protein,drugbinding protein.
\end{abstract}

\section{Introduction}

When treating oncology patients, we are often confronted with the fact that the same treatment for the same type of tumor may have different effects in different patients. One of the vital factors that determine the success of chemotherapy is the bioavailability of the drug. Drugs are bound to different proteins in blood plasma and only a free, unbound fraction is really active. There are, therefore, several plasma proteins that can affect the success of therapy and thus the overall outcome of treatment. One such plasma protein could also be alpha-1-acid glycoprotein.

Alpha-1-acid glycoprotein (AGP), also known as orosomucoid (ORM) is one of the most extensively studied plasma acute-phase proteins. AGP is a glycoprotein found in the alpha-1 fraction of plasma proteins. It was first described by Schmid (1) and Winzler et $\mathrm{al}(2)$. It is a protein with a very low isoelectric point $(\mathrm{pI}=2.8-3.8)$ and a high carbohydrate content (up to $45 \%$ ). Its molecular weight is about $37,000-54,000$ Da depending on the extent of glycosylation. The AGP molecule forms a single polypeptide chain composed of 183 amino acids. The main site of synthesis is the liver. The standard level of AGP for humans is 400-1200 mg. $1^{-1}$. AGP is considered to be a member of the acute-phase protein family.

${ }^{1}$ Institute of Medical Chemistry, Biochemistry and Clinical Biochemistry, Faculty of Medicine, Comenius University, Bratislava, Slovakia, and 23rd Department of Internal Medicine, University Hospital and Faculty of Medicine, Bratislava, Slovakia

Address for correspondence: L.Turecky, MD, PhD, Institute of Medical Chemistry, Biochemistry and Clinical Biochemistry, Faculty of Medicine, Comenius University Bratislava, Sasinkova 2, SK-81108 Bratislava, Slovakia.

Acknowledgements: This study was supported by the Scientific Grant Agency of the Ministry of Education, Slovak republic, VEGA No.1/0826/18.
Alpha-1-acid glycoprotein is an important representative of type 1 acute-phase proteins (type 1 acute-phase proteins are proteins regulated by IL-1, IL-6 and glucocorticoids). The human alpha-1-acid glycoprotein is the product of three adjacent genes $A G P-A, A G P-B$ and $A G P-B$ ' located on the long arm of chromosome 9 (3). AGP-A is expressed in the liver and encodes the variant of ORM1, which represents the major part of the serum AGP (about $75 \%$ of the total amount of AGP in the serum). The remaining two genes encode the ORM2 variant (4). Only AGP-A can be induced by acute-phase stimuli so that ORM1 can be considered as the only acute phase protein in the ORM family.

Although numerous articles have been devoted to AGP, its exact biological function remains obscure. Many biological properties of ORM have already been discussed in the existing literature: a) AGP is known as an acute-phase reactant and a disease marker, b) AGP is considered as a natural anti-inflammatory and immunomodulatory agent due to its anti-neutrophil and anti-complement activity (5) and the stimulation of the secretion of an IL-1 inhibitor by macrophages $(6,7), c)$ it is now generally accepted that AGP is an important drug-binding protein in the blood plasma as it binds both exogenous and endogenous ligands.

In our contribution we want to focus on the role of AGP in oncological patients.

\section{Changes of blood plasma levels of alpha-1-acid glycoprotein in cancer patients}

A systemic injury induces a significant rise in the hepatic synthesis of acute-phase proteins and therefore, the rise of synthesis of AGP. AGP concentration can rise up to 10 times higher in comparison to the reference range, depending on the severity of the disease state. Aberrant glycosylation of ORM suggests it is a 
strong candidate as a marker of the progression and prognosis of various types of cancer, with various glycoforms containing highly fucosylated tri- and tetra-antennary oligosaccharide side chains, which indicate a poor prognosis (8). It is known that plasma levels of orosomucoid are increased in several types of tumors.

\section{Gastrointestinal malignancies}

Several papers describe elevated values of AGP in patients with gastrointestinal tumors. There is data about increased ORM levels in patients with hepatocellular, cholangiocellular, colorectal and gastric carcinoma. Bachtiar et al (10) showed that AGP levels were significantly higher in hepatocellular carcinoma (HCC) patients than in chronic liver disease with $77 \%$ sensitivity and $83 \%$ accuracy. The results of Gani et al (11) showed that combination of AFP and AGP determination is superior to either marker alone in diagnosing $\mathrm{HCC}$ in liver cirrhosis patients. The combination of plasma AFP and AGP determination may be used to prompt an early diagnosis screening of hepatocellular carcinoma. Zhang et al (12) studied the glycosylation change of ORM in patients with $\mathrm{HCC}$ and with liver cirrhosis. The sialylation and fucosylation of AGP were different among hepatocellular carcinoma, liver cirrhosis and control group. The changes in glycosylation showed an excellent diagnostic ability to differentiate hepatocellular carcinoma from cirrhosis. Tanabe et al (13) described similar differences in the degree of glycosylation of AGP in patients with HCC and liver cirrhosis. They showed that AGP with multifucosylated tetraantennary $\mathrm{N}$-glycans was significantly elevated in hepatocellular carcinoma patients, whereas the single fucosylated derivative was not.

The cholangiocellular carcinoma is another malignant tumor of the liver. It is a tumor with relatively ineffective diagnosis. Rucksaken et al (14) observed overexpression of ORM2 in the cytoplasm of bile duct tumor cells and in the adjacent normal hepatocytes. The determination of plasma ORM2 levels showed significantly higher values in patients with cholangiocarcinoma than in the control group. The sensitivity and specificity of ORM2 in distinguishing cholangiocarcinoma patients from healthy persons were $92.86 \%$ and $73.68 \%$, respectively.

Colorectal cancer is the most commonly diagnosed gastrointestinal cancer. Yüceyar et al (15) studied AGP in combination with alpha-1-antitrypsin (AAT), C-reactive protein (CRP), carcinoembryonic antigen (CEA) and CA 19-9 at its preoperative staging. There was no correlation between sera CEA, AAT and CRP levels in relation to the stage of disease. There is a significant correlation between CA 19-9, AGP and the extent of disease. Yüceyar et al (15) believed that serum AGP levels in combination with serum CA 19-9 levels may have an important role in the preoperative staging of colorectal cancer. Gao et al (16) investigated ORM2 levels to validate the prognostic significance of plasma ORM2 in patients with colorectal cancer. The results showed that ORM2 levels in stage II of colorectal cancer patients were significantly higher than those of healthy controls. They found also association between plasma ORM2 level with local invasion of stage II of colorectal cancer and lymph node numbers. The patients with elevated plasma ORM2 had significantly shorter survival rates. These findings demonstrate that plasma ORM2 is an independent prognostic factor in patients with stage II of the colorectal cancer.

Ohbatake et al (17) reported elevated levels of AGP in sera and ascitic fluid of patients with gastric cancer in comparison to the healthy volunteers. Rashid et al (18) found out that after dividing patients with gastric cancer according to whether the AGP was within normal limits or elevated ones, the survival chance of the two groups was significantly different. The patients with normal AGP levels had a significantly longer survival rate than those with raised levels.

\section{Gynecological malignancies}

In the literature there are numerous contributions about ovarian cancer and AGP. In women with ovarian carcinoma a significant rise of orosomucoid was found, as compared with non-tumorous diseases with the exception of adnexitis $(19,20)$. Piver et al (21) in their study presented the results based on monitoring of fifty-six patients with ovarian carcinoma with serum AGP levels. According to their results the specificity of the AGP was $100 \%$, the sensitivity was only $12.5 \%$ and the overall accuracy $37 \%$. Fish et al (22) used the determination of serum alpha1-acid glycoprotein as an indicator of the effectiveness of cis-diamminedichloroplatinum in ovarian cancer patients. The results of their study showed that alpha-1-acid glycoprotein levels correlate with tumor burden before the therapy and the follow-up. Miyamoto et al (23) described aberrant glycosylation of alpha-1-acid glycoprotein in patients with ovarian cancer.

Patel et al (24) published the results of study of ORM in women with benign breast diseases and women with breast cancer. Orosomucoid was significantly elevated in untreated patients with breast cancer when compared with healthy women and patients who had benign breast diseases. A good correlation was observed between favorable treatment response and the decline in orosomucoid levels. AGP levels in patients who did not respond to anticancer therapy remained stable or increased during the follow-up.

\section{Hematological malignancies}

AGP was commonly increased in patients with multiple myeloma. There was no difference between the AGP levels in group of patients $>65$ years old and group of patients $<65$ years old (25). Alexandrakis et al (26) found that AGP is an independent prognostic factor for survival in patients with multiple myeloma.

AGP was studied also in lymphomas. The levels of AGP were increased in sera of experimental animals with lymphoma $(27,28)$ and also in patients with non-Hodgkin lymphomas (29). Serum AGP was higher at a diagnostic stage than after anti-neoplastic treatment. The treatment resulted in a gradual decrease in serum AGP concentrations after 4 weeks of treatment and these concentrations returned to values comparable with those of control healthy group by 12 weeks of treatment. Winkel et al (28) concluded that AGP may be a useful protein marker for monitoring of anti-neoplastic treatment in experimental animals with lymphoma. The AGP levels were increased also in patients with advanced Hodgkin's disease (30). These concentrations were significantly reduced by the administration of chemotherapy. They concluded that serum concentration of AGP can serve as a useful marker for the assessment of tumor activity in patients with advanced Hodgkin's disease. 


\section{Lung cancer}

The lung cancer is a leading cause of cancer death in both developed and developing countries. Charet et al (31) reported increased levels of AGP in the group of patients with lung cancer. There was no significant difference in pretreatment levels of AGP between non-small-cell carcinoma and a small-cell-carcinoma. When comparing the results of remission and progression, groups have confirmed that AGP drops with the remission or rises with the progression. Yildrim et al (32) also found increased levels of AGP in patients with primary lung cancer. The levels were increased both in the non-small-cell and the small-cell lung cancer. Ayyub et al (33) found increased levels of AGP in patients with the squamous cell carcinoma ( $2.93 \mathrm{~g} / \mathrm{l})$ and adenocarcinoma (2.39 $\mathrm{g} / \mathrm{l})$ when compared with the healthy controls $(0.83 \mathrm{~g} / \mathrm{l})$.

\section{Other malignancies}

AGP levels were increased also in patients with pheochromocytoma (34). The tumor removal led to a significant decrease in orosomucoid levels. Serum concentrations of bound sialic acid, free sialic acid and AGP in patients with laryngeal cancer were compared to those in serum specimens from the healthy controls. The mean levels of bound sialic acid and AGP were significantly increased in the laryngeal cancer patients versus the controls. There was no difference in serum free sialic acid levels between patients and the controls. As the stage of the cancer advanced, progressively higher levels of AGP were observed. The results indicate that serum AGP shows correlation with the stage of laryngeal carcinoma (35). The serum levels of AGP were investigated in patients with glioblastoma multiforme in relation to their prognosis. AGP was higher in patients who died within one year after admission than in those with a longer survival time. It is suggested that serum AGP profiles provide prognostic information in patients with glioblastoma multiforme (36). The study of Li et al (37) showed increased urinary levels of ORM1 in patients with bladder cancer. The correlation analysis indicated that urinary ORM1 had high positive correlation with the pathology classification of bladder cancer. Authors of this study concluded, that elevated urinary ORM1 could be a useful biomarker for bladder cancer.

\section{Alpha-1-acid glycoprotein as a drug-transporting and a drug-binding protein}

It is generally accepted that ORM is an important drug-binding and drug-transporting protein. Human plasma albumin and ORM are the most important drug-binding proteins that have important pharmacokinetic implications (38). In recent decades, drug-protein interactions have been widely studied and several methods of analysis of these phenomena have been developed and improved (39). The changes in AGP plasma concentrations occurring during various pathological states can considerably alter the free plasma drug level without affecting its total plasma concentration. Due to its physical-chemical properties, orosomucoid mainly binds basic and neutral drugs. It is generally assumed that in plasma, acidic drugs are mainly bound to albumin. However, binding to ORM will contribute significantly to the total plasma binding of these drugs, especially in diseases in which plasma level of ORM increases and/ or albumin level decreases (40). In addition, the existence of two forms of ORM in the blood plasma has also an influence on the drug-binding affinity. It was demonstrated that drugs like methadone, propafenone and amitryptiline bind selectively to ORM2, as opposed to warfarin, imatinib and dipyridamole, which prefer the ORM1 variant (41).

We assume that changes in serum AGP levels in patients with oncological diseases could result in a change in the pharmacokinetics of the administered drugs. Elevated serum AGP levels commonly observed in oncology patients and associated increased anti-cancer drug binding to AGP may result in the reduction in the free, biologically active fraction of the anti-cancer drug and thus lead to anti-tumor treatment failure.

Docetaxel is one of anticancer drugs with high affinity to alpha-1 acid glycoprotein. Docetaxel is an antitumor agent that belongs to the taxane family and is used in the treatment of various types of cancer (e.g. breast cancer, head and neck cancer, stomach cancer, prostate cancer and non-small-cell lung cancer) and neutropenia is a dose-limiting factor for its clinical use. AGP and albumin are the main carriers of docetaxel in plasma, and owing to the high inter-individual variability of AGP plasma concentrations, particularly in cancer, it was concluded that AGP should be the main determinant of docetaxel plasma binding variability. Drugs potentially co-administered with docetaxel (cisplatin, dexamethasone, doxorubicin, etoposide, vinblastine) did not modify the docetaxel plasma binding (42). Pharmacokinetic and pharmacodynamic analyses conducted during the development of docetaxel showed that patients with non-small-cell lung cancer with high baseline alpha-1-acid glycoprotein levels had a shorter time to progression and eventually to death. Kenmotsu et al (43) studied the effects of advanced age and serum AGP on docetaxel unbound exposure and neutropenia, dose-limiting toxicity, in cancer patients. The clearance of docetaxel and the degree of unbound fraction were significantly associated with serum AGP level, but not with age. Regardless of ageing, serum levels of AGP determine docetaxel unbound exposure and related dose-limiting toxicity. Serum AGP level and absolute neutrophil count at baseline appear to be predictive of neutropenia for patients of all ages following the administration of docetaxel. Similar results were also presented by Fukae et al (44). They showed in their study that albumin and AGP negatively correlated with the development of neutropenia and that the time course of neutrophil counts was predictable.

Imatinib (STI-571) is a potent inhibitor of tyrosine kinase activity of bcr/abl, an oncogenic fusion protein that causes chronic myelogenous leukemia (CML). Animal experiments showed that tumors regressed initially in all imatinib-treated mice, but all mice treated 15 days after the injection of tumor cells eventually relapsed. These animals did not respond to further imatinib treatment and their brc/abl kinase activity in vivo was not inhibited by imatinib, despite high plasma concentrations of the drug. However, the tumor cells from resistant animals were sensitive to imatinib in vitro, suggesting that a molecule in the plasma of relapsed animals may inactivate the drug. Plasma AGP bound to imatinib at physiological concentrations in vitro and blocked the ability of 
imatinib to inhibit bcr/abl kinase activity in a dose-dependent manner. Erythromycin competed with imatinib for AGP binding. When animals with large tumors were treated with imatinib alone or with a combination of imatinib and erythromycin, greater tumor reductions and better long-term tumor-free survival were observed after the combination treatment (45). They concluded that AGP binds to imatinib, preventing this compound from inhibiting the bcr/abl tyrosine kinase. Molecules such as erythromycin that compete with imatinib for binding to AGP may enhance the therapeutic potential of this drug. Gambacorti-Passerini et al (46) have undertaken a pharmacokinetic analysis of imatinib in chronic myelogenous leukemia. A positive correlation between AGP and imatinib plasma levels was observed. In vitro experiments using fresh blasts from CML patients showed, that AGP, at concentrations observed in the patients, decreased imatinib intracellular concentrations up to 10 times and blocked imatinib activity. The incubation with clindamycin restored imatinib intracellular concentrations and biological activity. Based on these results it can be concluded that AGP exerts significant effects on the pharmacokinetics, plasma concentrations and intracellular distribution of imatinib in CML patients. Zhong et al (47) studied the relationship between serum AGP, disease progression, and efficacy of imatinib treatment in CML patients. Serum AGP levels of no response group were significantly higher than those of a complete cytogenetic response, a complete hematologic response and the control group. Serum AGP levels of accelerated/blastic phase group were significantly higher than in complete cytogenetic response group. Suttorp et al (48) described similar effects of alpha-1-acid glycoprotein on pharmacokinetics of imatinib in pediatric patients.

Another anticancer drug that is known to bind to AGP is paclitaxel. Paclitaxel (PTX) is used to treat a number of types of cancer (e.g. ovarian cancer, breast cancer, lung cancer, Kaposi sarcoma, cervical cancer and pancreatic cancer). Paclitaxel is often used to treat gastric cancer patients with peritoneal carcinomatosis because it can disseminate easily, with a high rate of passage from the systemic circulation into the peritoneal cavity. Drugs in peripheral circulation are present as free compounds or bound to plasma proteins. In general, only the free fraction has pharmacodynamics activity because only free unbound drug can diffuse across biological barriers and be transported to their sites of action. A large fraction of PTX binds to AGP. It is known that AGP concentrations are increased in cancer patients. These findings suggested that increased AGP concentrations in gastric cancer patients reduce the unbound fraction of PTX and suppress the activity of PTX. The study of Ohbatake et al (17) sought to clarify whether AGP binds to PTX and alters its anticancer effects. The mean AGP concentrations in the serum and ascites of gastric cancer patients were higher than the mean AGP concentration observed in the sera of the healthy controls. Increased AGP concentrations significantly suppressed the cell growth inhibitory effect of PTX in vitro, but the co-administration of erythromycin restored it. Authors of this study showed that AGP is an important regulatory factor modulating the anticancer activity of intraperitoneal PTX. The co-administration of PTX and erythromycin may be effective in treating gastric cancer patients with peritoneal carcinomatosis.
Trabectedin and its analogue lurbinectedin are effective drugs used in the treatment of ovarian cancer. Erba et al (49) described the possibility that AGP present in ascites of patients with ovarian cancer might reduce the activity of lurbinectedin and trabectedin.

\section{Immuno-modulating effects of alpha-1-acid glycoprotein}

The importance of influencing the immune system in the treatment of cancer is also confirmed by the fact that two important immunologists J.P. Allison and T. Honjo were awarded the Nobel Prize in Medicine in 2018 for their discovery of cancer therapy by inhibition of negative immune regulation (50).

Orosomucoid has been found to have immune-modulatory effects, such as inhibiting leukocyte rolling/adhesion and migration in vivo, and the chemotaxis response in vitro (51). It can decrease neutrophil chemotaxis, migration, and superoxide production, which consequently increases susceptibility to sepsis in diabetic mice (52), ORM1 can reversible inhibit the proliferation of lymphocytes while decreasing IL-2 synthesis (53). Human AGP was found to significantly inhibit the proliferation response of human peripheral blood lymphocytes to phytohemagglutinin. The inhibitory effect of AGP was at the level of both the lymphocyte and the mitogen. AGP also inhibited blastogenesis induced by ConA. The inhibitory effect of AGP was directed mainly against T-cells and not B-cells (54).

Inhibition of lymphocyte proliferation by AGP was demonstrated to correlate with a decrease in interleukin IL-2 synthesis by the lymphocytes (55). All these experiments required doses of AGP that were in the range of normal or inflamed serum levels. It has been hypothesized that this nonspecific immunosuppressive activity of AGP can be a major problem in cancer patients because an impaired immune defense against the tumor may result in immune escape of the tumor (55). In contrast, AGP has also been shown to inhibit growth of several types of tumor cells in vitro (56). Impairment of cellular immune responses to tumor elimination is common in hosts bearing progressively growing malignant tumors. The lymphocytes from tumor-bearing hosts are hypo-responsive to various mitogenic and antigenic stimuli. The immunosuppression has been attributed to the presence of a variety of soluble factors such as tumor antigens, immune complexes and other immune-regulatory proteins (57). One of these serum immunosuppressive factors could be AGP, the level of which is significantly increased in tumor disease.

\section{Pro-angiogenic properties of orosomucoid}

Another potential function of orosomucoid is its participation in the regulation of angiogenesis. Angiogenesis is the physiological process through which new blood vessels form from pre-existing vessels. Angiogenesis is a normal and vital process in growth and development. However, it is also a fundamental step in the transition of tumors from a benign to a malignant state. Chemical regulation of angiogenesis is performed by various angiogenic activators and inhibitors. Among these factors VEGF is the key regulator of physiological and pathological angiogenesis and acts as a survival factor for endothelial cells. Tumors induce blood 
vessel growth (angiogenesis) by secreting various growth factors (e.g. VEGF). Growth factors such as FGF and VEGF can induce capillary growth into the tumor. Angiogenesis is also required for the spread of tumor, or metastasis. Irmak et al (56) analyzed the potential role of ORM in angiogenesis. They showed that ORM increases endothelial cell migration in a dose dependent manner and supports the VEGF-A induced endothelial tube formation in vitro when VEGF and ORM are applied simultaneously. While in vitro ORM alone does not induce endothelial tubes, it increases the number of blood vessels in vivo in chorioallantoic membrane tissue. Also in chorioallantoic membrane assay ORM enhances the VEGF-A -induced new vessel formation. These data suggest for the first time that ORM is involved in angiogenesis and supports VEGF-A mediated new vessel formation. The results of this study suggest that ORM produced by endothelial cells acts in an autocrine and the circulating ORM in paracrine manner on vascular endothelial cells. ORM is essentially involved in the regulation of angiogenesis, particularly in the presence of VEGF-A, and with these, pro-angiogenic properties ORM may play a role in tumor vascularization.

\section{Conclusion}

We can conclude that changes in plasma levels of alpha-1-acid glycoprotein could affect the overall outcome of treatment of a cancer patient. Some chemotherapeutics significantly bind to orosomucoid and their free fraction is dependent on the concentration of this protein in the blood. Elevated levels of orosomucoid found in most cancer patients can cause increased drug binding to the protein and reduce the free, biologically active fraction. The higher the plasma level of orosomucoid, the lower the level of the bioavailable drug, which may cause treatment failure. When using antitumor drugs that bind to orosomucoid, it is essential to know the plasma level of this protein, and if there is a significant increase, co-administer any other drug that competitively displaces the antitumor drug from the orosomucoid binding and thereby increase the efficacy of the treatment. High levels of orosomucoid may affect the outcome of treatment of tumor disease also due to its immunomodulatory effect and the ability to affect angiogenesis.

\section{References}

1. Schmid K. Preparation and properties of an acid glycoprotein prepared from human plasma. J Am Chem Soc 1950; 72: 2816.

2. Weimer HE, Mehl JW, Winzler RJ. Studies on the mucoproteins of human plasma. V.Isolation and characterization of a homogenous mucoprotein. J Biol Chem 1950; 185 (2): 561-568.

3. Umetsu K, Yuasa I, Harada A et al. Orosomucoid phenotyping with monoclonal antibodies: polymorphic occurrence of ORM $1 * \mathrm{Q} 0$ in aboriginal Taiwanese populations. Hum Hered 1995; 45: 181-185.

4. Dente L, Pizza MG, Metspalu A, Cortese R. Structure and expression of the genes coding for human alpha 1-acid glycoprotein. EMBO J 1987; 6 (8): 2289-2296.

5. Williams JP, Weiser MR, Pechet TT, Kobzik L, Moore Jr FD, Hechtman HB. Alpha 1-acid glycoprotein reduces local and remote injuries after intestinal ischemia in the rat. Am J Physiol 1997; 273: G1031-G1035.
6. Bories PN, Feger J, Benbernou N, Rouzeau JD, Agneray J, Durand G. Prevalence of tri- and teraantennary glycans of human alpha 1-acid glycoprotein in release of macrophage inhibitor of interleukin-1 activity. Inflammation 1990; 14 (3): 315-323.

7. Tilg H, Vannier E, Vachino G, Dinarello C, Mier JJW. Antiinflammatory properties of hepatic acute phase proteins: preferential induction of interleukin 1 (IL-1) receptor antagonist over IL-1 beta synthesis by human peripheral blood mononuclear cells. J Exp Med 1993; 178 (5): 1629-1636.

8. Hashimoto S, Asao T, Takahashi J et al Alpha 1-acid glycoprotein fucosylation as a marker of carcinoma progression and prognosis. Cancer 2004; 101 (12): 2825-2836.

9. Yazawa S, Yokobori T, Kaira K, Kuwano H, Asao T. A new enzyme immunoassay for the determination of highly sialylated and fucosylated human alpha-1-acid glycoprotein as a biomarker of tumorigenesis. Clin Chim Acta 2018; 478: 120-128.

10. Bachtiar I, Santoso JM, Atmanegara B et al. Combination of alpha1 -acid glycoprotein and alpha-fetoprotein as an improved diagnostic tool for hepatocellular carcinoma. Clin Chim Acta 2009; 399 (1-2): 97-101.

11. Gani RA, Suryamin M, Hasan I, Lesmana CR, Sanityoso A. Performance of alpha-fetoprotein in combination with alpha 1-acid glycoprotein for diagnosis of hepatocellular carcinoma among liver cirrhosis patients. Acta Med Indones 2015; 47 (3): 216-222.

12. Zhang D, Huang J, Luo D, Feng X, Liu Y, Liu Y. Glycosylation as a serum biomarker for hepatocellular carcinoma and cirrhosis. Biomark Med 2017; 11 (5): 423-430.

13. Tanabe K, Kitagawa K, Kojima N, Iijima S. Multifucosylated alpha1 -acid glycoprotein as a novel marker for hepatocellular carcinoma. J Proteome Res 2016; 15 (9: 2935-2944.

14. Rucksaken R, Charoensuk L, Pinlaor P, Pairojkul C, Khuntikeo N, Pinlaor S. Plasma orosomucoid 2 as a potential risk marker of cholangiocarcinoma. Cancer Biomark 2017; 18 (1): 27-34.

15. Yuceyar S, Ertürk S, Dirican A, Cengiz A, Saner H. The role of acutephase reactant proteins, carcinoembryonic antigen and CA 19-9 as a marker in the preoperative staging og colorectal cancer: a prospective clinical study. Int Surg 1996; 81 (2): 136-139.

16. Gao F, Zhang $\mathrm{X}$, Whang $\mathrm{S}$, Zheng $\mathrm{Ch}$. Prognostic impact of plasma ORM2 levels in patients with stage II colorectal cancer. Ann Clin Lab Sci 2014; 44 (4): 388-393.

17. Ohbataka Y, Fushida S, Tsukada T et al. Elevated alpha 1-acid glycoprotein in gastric cancer patients inhibits the anticancer effects of paclitaxel, effects restored by co-administration of erythromycin. Clin Exp Med 2016; 16: 585-592.

18. Rashid SA, O' Quigley J, Axon AT, Cooper EH. Plasma protein profiles and prognosis in gastric cancer. Br J Cancer 1982; 45 (3): 390-394.

19. Tošner J, Krejsek J, Louda B. Využití orosomukoidu a prealbuminu k diferenciální diagnose onemocnění vaječníku. Čs Gynekol 1988; 53 (5): 370-374.

20. Katnik I, Gerber J, Dobryszycka W. Microheterogeneity of alpha 1-acid glycoprotein in the sera of patients with cancer or inflammatory states of the ovaries. Arch Immunol Ther Exp (Warsz) 1988; 36 (1): 1-6.

21. Piver MS, Mayer M, Diakun K, Lele SB, Chu TM. Serum alpha 1-acid glycoprotein in epithelial ovarian cancer. Gynecol Oncol 1988; 29 (3): 305-308.

22. Fish RG, Gill TS, Adams M, Kerby I. Serum haptoglobin and alpha 1 -acid glycoprotein as indicators of the effectiveness of cis-diamminedichloroplatinum (CDDP) in ovarian cancer patients - a preliminary report. Eur J Cancer Clin Oncol 1984; 20 (5): 625-630. 
23. Miyamoto S, Stroble CD, Taylor S et al. Multiple reaction monitoring for the quantitation of serum protein glycosylation profiles: application to ovarian cancer. J Proteome Res 2018; 17 (1): 222-233.

24. Patel PS, Patel MM, Raval GN, Rawal RM, Balar DB, Patel DD. Seromucoid fraction: a useful biomarker for patients with breast cancer. Am J Clin Oncol 1998; 21 (3): 258-262.

25. Dupire S, Wemeau M, Debarri H et al. Prognostic value of PINI index in patients with multiple myeloma. Eur J Haematol 2012; 88 (4): 306-313.

26. Alexandrakis MG, Passam FH, Ganotakis ES, Sfiridaki K, Xilouri I, Perisinakis K, Kyriakou DS. The clinical and prognostic significance of erythrocyte sedimentation rate, serum interleukin- 6 and acute protein levels in multiple myeloma. Clin Lab Haematol 2003; 25 (1): 41-46.

27. Hahn KA, Freeman KP, Barnhill MA, Stephen EL. Serum alpha1acid glycoprotein concentrations before and after relapse in dogs with lymphoma treated with doxorubicin. J Am Vet Med Assoc 1999; 214 (7): $1023-1025$.

28. Winkel VM, Pavan TL, Wirthl VA, Alves AL, Lucas SR. Serum alpha 1 acid glycoprotein and serum amyloid A concentrations in cats receiving antineoplastic treatment for lymphoma. Am J Vet Res 2015; 76 (11): 983-988.

29. Martinsson U, Glimelius B, Hagberg H, Sundström C. Prognostic relevance of serum-markers in relation to histopathology, stage and initial symptoms in advanced low-grade non-Hodgkin's lymphomas. Eur J Haematol 1988; 40 (4): 289-298

30. Zielinski CC, Preis P, Aiginger P, Eibl MM. Acute-phase proteins and parameters of humoral immunity in patients with advanced Hodgkin's disease. J Cancer Res Clin Oncol 1985; 110 (1): 65-70.

31. Charet JC, Watine J, Lepretre A, Raimbault C, Charet P. Orosomucoid:prealbumin ratio in the monitoring of lung cancer. Clin Biochem 1996; 29 (3): 287-290

32. Yildirim A, Meral M, Kaynar H, Polat H, Ucar EY. Relationship between serum levels of some acute-phase proteins and stage of disease and performance status in patients with lung cancer. Med Sci Monit 2007; 13 (4): 195-200.

33. Ayyub A, Saleem M, Fatima I, Tariq A, Hashmi N, Musharraf SG. Glycosylated alpha-1-acid glycoprotein 1 as a potential lung cancer serum biomarker. Int J Biochem Cell Biol 2016; 70: 68-75.

34. Zelinka T, Petrák $\mathbf{O}$, Strauch B et al. Elevated inflammation markers in pheochromocytoma compared to other forms of hypertension. Neuroimmunomodulation 2007; 14 (1): 57-64.

35. Uslu C, Taysi S, Akcay F, Sutbeyaz MY, Bakan N. Serum free and bound sialic acid and alpha-1-acid glycoprotein in patients with laryngeal cancer. Ann Clin Lab Sci 2003; 33 (2): 156-159.

36. Matsuura H, Nakazawa S. Prognostic significance of serum alpha 1 -acid glycoprotein in patients with glioblastoma multiforme: a preliminary communication. J Neurol Neurosurg Psychiatry 1985; 48 (8): 835-837.

37. Li F, Yu Z, Chen P et al. The increased excretion of urinary orosomucoid 1 as a useful biomarker for bladder cancer. Am J Cancer Res 2016; 6 (2): $331-340$

38. Dalhoff A. Seventy-five years of research on protein binding. Antimicrob Agents Chemother 2018; 62 (2): e01663-17. doi: 10.1128/AAC.02317-17.

39. Wanat K, Brzezinska E, Sobanska AW. Aspects of drug-protein binding and methods of analyzing the phenomenon. Curr Pharm Design 2018. doi: $10.2174 / 1381612824666180808145320$.

40. Urien S, Albengres E, Pinquier JL, Tillement JP. Role of alpha-1 acid glycoprotein, albumin and nonesterified fatty acids in serum binding of apazone and warfarin. Clin Pharmacol Ther 1986; 39 (6): 683-689.
41. Luo Z, Lei H, Sun Y, Liu X, Su DF. Orosomucoid, an acute response protein with multiple modulating activities. J Physiol Biochem 2015; 71 : 329-340.

42. Urien S, Barré J, Morin C, Paccaly A, Montay G, Tillement JP. Docetaxel serum protein binding with high affinity to alpha 1-acid glycoprotein. Invest New Drugs 1996; 14 (2), 147-151.

43. Kenmotsu H, Imamura CK, Ono A et al. The effect of advanced age and serum alpha-1-acid glycoprotein on docetaxel unbound exposure and dose-limiting toxicity in cancer patients. Br J Clin Pharmacol 2017; 83 (11): 2416-2425.

44. Fukae M, Shiraishi Y, Hirota T et al. Population pharmacokinetic-pharmacodynamic modeling and model-based prediction of docetaxel-induced neutropenia in Japanese patients with non-small cell lung cancer. Cancer Chemother Pharmacol 2016; 78 (5): 1013-1023.

45. Gambacorti-Passerini C, Barni R, le Coutre $\mathbf{P}$ et al. Role of alpha 1 acid glycoprotein in the in vivo resistance of human BCR-ABL $(+)$ leukemic cells to the abl inhibitor STI571. J Natl Cancer Inst 2000; 92 (20): 1641-1650.

46. Gambacorti-Passerini C, Zucchetti M, Russo D et al. Alpha 1 acid glycoprotein binds to imatinib (STI571) and substantially alters its pharmacokinetics in chronic myeloid leukemia patients. Clin Cancer Res 2003; 9 (2): 625-632.

47. Zhong JS, Meng FY, Xu D, Dai M, Wei YQ, Zhou HS. Serum alpha1acid glycoprotein, imatinib concentration and efficacy in chronic myeloid leukemia patients. Zhonghua Yi Xue Za Zhi 2011; 91 (30): 2120-2123.

48. Suttorp M, Bornhäuser M, Metzler M, Millot F, Schleyer F. Pharmacology and pharmacokinetics of imatinib in pediatric patients. Expert Rev Clin Pharmacol 2018; 11 (3): 219-231.

49. Erba E, Romano M, Gobbi M et al. Ascites interferes with the activity of lurbinectedin and trabectedin: Potential role of their binding to alpha1-acid glycoprotein. Biochem Pharmacol 2017; 144: 52-62.

50. Bernadic M Jr, Duchon R, Aziri R, Mladosievicova B. New principles of cancer therapy give new hope for oncological patients. Nobel Prize in Medicine 2018. Bratisl Med J 2019; 120 (1): 15-18.

51. Mestriner FL, Spiller F, Laure HJ et al. Acute-phase protein alpha-1-acid glycoprotein mediates neutrophil migration failure in sepsis by a nitric oxidedependent mechanism. Proc Natl Acad Sci USA 2007; 104: 19595-19600.

52. Spiller F, Carlos D, Souto FO et al. Alpha 1-acid glycoprotein decreases neutrophil migration and induces susceptibility to sepsis in diabetic mice. Diabetes 2012; 61 (6): 1584-1591.

53. Elg SA, Mayer AR, Carson LF, Twiggs LB, Hill RB, Ramakrishnan S. Alpha-1-acid glycoprotein is an immunosuppressive factor found in ascites from ovaria carcinoma. Cancer 1997; 80 (8): 1448-1456.

54. Hochepied T, Berger FG, Baumann H, Libert C. Alpha 1-acid glycoprotein: an acute phase protein with inflammatory and immunomodulating properties. Cytokine Growth Factor Rev 2003; 14: 25-34.

55. Samak R, Edelstein R, Israel L. Immunosuppressive effect of acutephase reactant proteins in vitro and its relevance to cancer. Cancer Immunol Immunother 1982; 13: 38-43.

56. Shibata Y, Tamura K, Ishida N. In vivo analysis of the suppressive effects of immunosuppressive acidic protein, a type of alpha-1-acid glycoprotein, in connection with its high level in tumor-bearing mice. Cancer Res 1983; 43: 2889-2896.

57. Irmak S, Oliveira-Ferrer L, Singer BB, Ergün S, Tilki D. Pro-angiogenic properties of orosomucoid (ORM). Exp Cell Res 2009; 315 (18): 3201-3209.

Received August 1, 2018. Accepted September 3, 2018. 